\title{
A TRANVERSALITY THEOREM FOR HOLOMORPHIC MAPPINGS AND STABILITY OF EISENMAN-KOBAYASHI MEASURES
}

\author{
SH. KALIMAN AND M. ZAIDENBERG
}

\begin{abstract}
We show that Thom's Transversality Theorem is valid for holomorphic mappings from Stein manifolds. More precisely, given such a mapping $f: S \rightarrow M$ from a Stein manifold $S$ to a complex manifold $M$ and given an analytic subset $A$ of the jet space $J^{k}(S, M), f$ can be approximated in neighborhoods of compacts by holomorphic mappings whose $k$-jet extensions are transversal to $A$. As an application the stability of Eisenman-Kobayshi intrinsic $k$-measures with respect to deleting analytic subsets of codimension $>k$ is proven. This is a generalization of the Campbell-Howard-Ochiai-Ogawa theorem on stability of Kobayashi pseudodistances.
\end{abstract}

\section{INTRODUCTION}

1.1. Let $X$ and $M$ be connected complex manifolds. Denote by $\operatorname{Hol}(X, M)$ the space of holomorphic mappings $X \rightarrow M$ and by $J^{k}(X, M)$ the space of $k$-jets of holomorphic mappings $X \rightarrow M$. We say that $A \subset J^{k}(X, M)$ is a stratified analytic subset if $A$ is a closed analytic subset in $J^{k}(X, M)$ (regarded as a complex manifold) with a stratification which satisfies Whitney's condition (a). Recall that for every

closed analytic subset such stratification always exists [W]. The symbol $j_{k}(f) \pitchfork A$ where $f \in \operatorname{Hol}(X, M)$ means that the $k$-jet extension $j_{k}(f): X \rightarrow J^{k}(X, M)$ of $f$ is transversal to each stratum of the given stratification of $A$.

The main result of this paper is the following analytic version of Thom's Transversality Theorem $[\mathrm{T}]$. (To our surprise we did not find this fact in the literature, and it must be formulated.)

1.2. Transversality Theorem. Let $X$ and $M$ be as above. Suppose that $X$ is Stein and $M$ is endowed with a Hermitian metric $h$. Let $A_{i} \subset J^{k_{i}}(X, M)$ be a sequence of stratified analytic subsets where $k_{i}$ is a nonnegative integer and $i=$ $1,2, \ldots$. Let $f \in \operatorname{Hol}(X, M)$ and let $Y$ be a closed analytic subset of $X$ such that $\left.j^{k_{i}}(f)\right|_{Y} \pitchfork A_{i}$ for every $i$ (where the restriction is regarded as a mapping from $Y$ to $\left.J^{k_{i}}(X, M)\right)$. Then for each compact subset $K \subset X$, every natural $k$, and every $\varepsilon>0$ there exist an open neighborhood $\Omega$ of $K$ in $X$ and a holomorphic mapping $\tilde{f}=f_{\varepsilon, K, k}: \Omega \rightarrow M$ such that

(1) $j^{k_{i}}(\tilde{f}) \pitchfork A_{i}, \quad i=1,2, \ldots$;

Received by the editors November 16, 1994.

1991 Mathematics Subject Classification. Primary 32E10, 32H02, 58C10, 58A35, 58A07.

Supported by General Research Support Award. 
(2) $\left.j^{k}(\tilde{f})\right|_{Y \cap \Omega}=\left.j^{k}(f)\right|_{Y \cap \Omega}$;

(3) $\operatorname{dist}_{h}\left(\left.f\right|_{\Omega}, \tilde{f}\right)<\varepsilon$ uniformly in $\Omega$.

In the algebraic setting this theorem can be strengthened due to the DemaillyLempert-Shiffman Approximation Theorem [DLS].

1.3. Corollary. Let the assumption of Theorem 1.2 hold. Suppose also that $X$ is an affine algebraic variety, $M$ is a smooth quasiprojective variety, $Y$ is a closed algebraic subvariety of $X$, and for some $k>0$ the mapping $\left.j^{k}(f)\right|_{Y}: Y \rightarrow J^{k}(X, M)$ is a regular mapping. Then for each compact $K \subset X$, every $\varepsilon>0$, and every natural $n$ there exist a neighborhood $\Omega$ of $K$ in $X$ and a Nash algebraic mapping $\tilde{f}: \Omega \rightarrow M$ so that conditions (1) for $i=1, \ldots, n,(2)$, (3) from Theorem 1.2 hold.

Recall that $\tilde{f}$ being Nash algebraic means that the graph of $\tilde{f}$ is contained in an algebraic subvariety $N \subset X \times M$ where $\operatorname{dim} N=\operatorname{dim} X$; the same holds for the image of $\tilde{f}$.

1.4. Let $M$ be a connected complex manifold of complex dimension $m>0$. Then for any $k=1, \ldots, m$ the Eisenman $k$-measure $E_{M}^{k}$ is intrinsicly defined on the set of decomposable $k$-wedge vectors in $\Lambda^{k} T M$ and it has the following basic properties:

-every mapping of the $k$-wedge vector bundles of complex manifolds generated by a holomorphic mapping does not increase Eisenman measures; in $\mathbb{C}^{k}$;

- $E_{M}^{k}$ coincides with the Bergman volume in the case when $M$ is the unit ball $B^{k}$

Moreover, the Eisenman $k$-measure is the largest measure on decomposable $k$ wedge vectors with the two above properties. Further information on Eisenman measures and their applications may be found in [E], [P], [Ko 1], [Ko 2], [LZ], [PS], [GW], [Ka 2]. Mention also that $E_{M}^{1}$ is nothing but the Kobayashi-Royden infinitesimal pseudometric $K_{M}$ on $T M$, and $E_{M}^{m}$ is the Eisenman-Kobayashi pseudovolume form on $\Lambda^{m} T M$.

By Campbell-Howard-Ochiai-Ogawa's theorem [CHO], [CO] the Kobayashi pseudodistance $k_{M}$ of $M$ does not change under deleting analytic subsets of $M$ of codimension at least 2 . From the Transversality Theorem 1.2 we obtain a generalization of this result.

1.5. Corollary. Let $A$ be a closed analytic subset of $M$ of codimension at least $k+1$. Then $E_{M \backslash A}^{k}=\left.E_{M}^{k}\right|_{M \backslash A}$. In particular, for $k=1$ we have $K_{M \backslash A}=\left.K_{M}\right|_{M \backslash A}$ if $\operatorname{codim}_{M} A \geq 2$.

By Royden's Theorem [Ro] the Kobayashi pseudodistance $k_{M}$ is the integrated form of $K_{M}$. Therefore, $k_{M \backslash A}=\left.k_{M}\right|_{M \backslash A}$ if $\operatorname{codim}_{M} A \geq 2$ which is the CampbellHoward-Ochiai-Ogawa Stability Theorem.

1.6. It is easily seen that the Eisenman-Kobayashi volume of the unit ball does change after deleting the origin (see $[\mathrm{Ra}]$ for qualified estimates). Thus in general condition $\operatorname{codim}_{M} A \geq k+1$ of Corollary 1.5 cannot be made weaker. However, by a result of [Ka 1] if $A$ is a smooth closed submanifold of $M$ of codimension $\ell \geq 2$ then in some cases deleting $A$ affects the Eisenman $k$-measure $E_{M}^{k}$ exactly in the same way as blowing up $\sigma_{A}: M_{A} \rightarrow M$ of $M$ with centrum at $A$. More precisely, if either $\ell \geq\left[\frac{k-1}{2}\right]+2$ or the $(\ell-1)$-st Chern class of the normal bundle of $A$ is trivial then $\sigma_{A}^{*} E_{M_{A} \backslash A}^{k}=E_{M_{A}}^{k} \mid M_{A} \backslash E$ where $E$ is the exceptional divisor of $\sigma_{A}$ in $M_{A}$. In other words, the Eisenman $k$-measure does not change under deleting of a 
smooth divisor which can be contracted onto a smooth submanifold of codimension $\geq\left[\frac{k-1}{2}\right]+2$. In particular, deleting of the exceptional divisor of a blow-up with centrum at a point does not affect any of Eisenman $k$-measures. This stability result was a motivation for the present paper.

1.7. Returning to the Transversality Theorem note that in general an approximation of a given holomorphic mapping from a Stein manifold $X$ by transversal ones (which does exists in neighborhoods of compacts, by Theorem 1.2) cannot be found on the whole $X$ due to certain rigidity phenomena. This can be shown by simple examples.

1.8. Examples. a) Consider a smooth complex surface $S$ with a $(-1)$-curve $E \cong$ $\mathbb{P}^{1}$. Let $f: \mathbb{C} \rightarrow \mathbb{P}^{1} \cong E \hookrightarrow S$ be an embedding. Put $A=f(0) \in S$. Then $f$ cannot be uniformly approximated by holomorphic mappings $\tilde{f}: \mathbb{C} \rightarrow S$ transversal to $A$, since after blowing $E$ down the image of $\tilde{f}(\mathbb{C})$ would be contained in a ball with center at the image of $E$, but not coincide with this point. Thus, $\tilde{f}$ itself must be constant which is absurd. Moreover, if $S$ is a blow-up of a hyperbolic surface (for instance, $S$ is the blow-up of the unit ball $B^{2}$ in $\mathbb{C}^{2}$ at the origin) then the above $f$ cannot be approximated (not necessarily uniformly) on compacts by $\tilde{f} \in \operatorname{Hol}(\mathbb{C}, S)$ transversal to $A$, by the same reason.

b) Let $\pi: S \rightarrow C$ be a morphism of a smooth projective surface $S^{\prime}$ onto a curve $C$ whose generic fibers are pairwise non-isomorphic curves of genus $g \geq 2$. Fix a generic fiber $F=\pi^{-1}\left(c_{0}\right)$ over a point $c_{0} \in C$ and two distinct points $A, B \in F$. Put $R=F-\{B\}$. Then $R$ is an open Riemann surface, i.e. it is Stein. The identical embedding $f: R \hookrightarrow F \hookrightarrow S$ cannot be approximated uniformly by holomorphic mappings $\tilde{f}: R \rightarrow S$ transversal to $A$. Indeed, $\pi \circ \tilde{f} \equiv$ const $=c \neq c_{0}$ and, therefore, $\tilde{f}(R) \subset F_{c}=\pi^{-1}(c)$. Since $c$ can be assumed to be sufficiently close to $c_{0}$ the fiber $F_{c}$ is a hyperbolic curve which is not isomorphic to $F$. But, by the big Picard theorem, the mapping $\tilde{f}: R \rightarrow F_{c}$ can be extended to an isomorphism $F \rightarrow F_{c}$ which is impossible.

c) Consider the Stein domain $D=\left\{z=(x, y) \in \mathbb{C}^{2}|| x|<1| x y \mid,<1\right\}$. Clearly, the image of every non-constant holomorphic mapping $\mathbb{C} \rightarrow D$ is contained in the $y$-axis. Hence the identical embedding of this axis into $D$ cannot be approximated on compacts by holomorphic mappings $\mathbb{C} \rightarrow D$ transversal to the origin.

d) In $[\mathrm{BG}]$ a family of surfaces in $\mathbb{P}^{3}$ was constructed which gives a smooth proper morphism $\pi: M \rightarrow \Delta$ of a threefold $M$ onto the unit disc so that all fibers of $\pi$ except for the central one $F_{0}=\pi^{-1}(0)$ are hyperbolic, and $F_{0}$ is a Fermat surface of degree 50. It is known $[\mathrm{G}]$ that any entire curve $f: \mathbb{C} \rightarrow F_{0}, f \not \equiv$ const, is contained in a finite union of projective lines in $F_{0}$. Thus the same is true for every entire curve in $M$ due to the Liouville theorem and the hyperbolicity of nonzero fibers. Therefore, none of these curves can be pushed away from a point on it by means of small deformations.

\section{Preliminaries}

Let, as above, $X$ and $M$ be connected complex manifolds. By $J_{x}^{k}(X, M)$ we denote the stalk at $x$ of $k$-jets of holomorphic mappings $X \rightarrow M$. If $V$ is an open subset in $X$ then the jet space $J^{k}(V, M)$ can be viewed as a domain in $J^{k}(X, M)$. For a holomorphic vector bundle $\zeta=(T, \pi, X)$ we treat $k$-jets of its holomorphic sections as $k$-jets of holomorphic mappings $X \rightarrow T$. 
Let $A$ be a closed analytic subset of a complex manifold $N$ and $\Sigma$ be an analytic stratification of $A$ which satisfies Whitney's condition (a). By $A_{\Sigma, l}$ we denote the union of all strata of $\Sigma$ of dimension $\leq l$. It is known that $A_{\Sigma, l}$ is a closed analytic subset of $N$. By Whitney's condition (a), if $f \in \operatorname{Hol}(X, N)$ is transversal to $A_{\Sigma, l}$ then $f$ is transversal to $A$ in a neighborhood of $A_{\Sigma, l}$ as well as any $\tilde{f} \in \operatorname{Hol}(X, N)$ which is sufficiently uniformly close to $f$ in $C^{1}(X, N)$-topology.

\section{SOME LEMMAS ON HOLOMORPHIC VECTOR FIELDS AND COLLECTIVE TRANSVERSALITY}

As in the smooth case the proof of the Transversality Theorem is based on the Collective Transversality Lemma (see $[\mathrm{T}],[\mathrm{AVG}]$ ) which is the main result of this section. To prove it we need several simple lemmas on holomorphic vector fields. In the first of them we show that fixing the $k$-jet of a vector field at a point yields fixing the $k$-jet of the associated phase flow at this point.

3.1. Lemma. Let $\nu$ be a germ of a holomorphic vector field at the origin $\overline{0}_{r} \in \mathbb{C}^{r}$ with the phase flow $\varphi_{\nu, t}$, where $t$ is the complex time in a neighborhood of the origin in $\mathbb{C}$. If $j^{k}(\nu)\left(\overline{0}_{r}\right)=\overline{0}$ for some $k \geq 0$ then for any $t$ with $|t| \ll 1$ one has $j^{k}\left(\varphi_{\nu, t}\right)\left(\overline{0}_{r}\right)=j^{k}\left(\operatorname{id}_{\mathbb{C}^{r}}\right)\left(\overline{0}_{r}\right)$.

Proof. Since the phase flow $\varphi_{\nu, t}:\left(\mathbb{C}^{r} \times \mathbb{C}, \overline{0}_{r} \times 0\right) \rightarrow\left(\mathbb{C}^{r}, \overline{0}_{r}\right)$ is holomorphic, it has a convergent power series expansion

$$
\varphi_{\nu, t}(\bar{x})=\bar{x}+t \nu(\bar{x})+\sum_{i \geq 2} t^{i} \nu_{i}(\bar{x})
$$

in some neighborhood of the origin $\overline{0}_{r} \times 0 \in \mathbb{C}^{r} \times \mathbb{C}$, where $\bar{x} \in \mathbb{C}^{r}$ and $\nu_{i}(\bar{x}), i=$ $2, \ldots$, are germs of holomorphic vector fields at $\overline{0}_{r} \in \mathbb{C}^{r}$. Therefore,

$$
j^{k}\left(\varphi_{\nu, t}\right)\left(\bar{x}^{\prime}\right)=j^{k}\left(\operatorname{id}_{\mathbb{C}^{r}}\right)\left(\bar{x}^{\prime}\right)+t j^{k}(\nu)\left(\bar{x}^{\prime}\right)+\sum_{i \geq 2} t^{i} j^{k}\left(\nu_{i}\right)\left(\bar{x}^{\prime}\right) .
$$

Thus we have to show that the condition $j^{k}(\nu)\left(\overline{0}_{r}\right)=\overline{0}$ implies that $j^{k}\left(\nu_{i}\right)\left(\overline{0}_{r}\right)=\overline{0}$ for all $i \geq 2$. Note that $\varphi_{\nu, t+s}=\varphi_{\nu, s} \circ \varphi_{\nu, t}$ when $|s|$ and $|t|$ are small enough. Put $\nu_{1}(\bar{x})=\nu(\bar{x})$ and compare the two convergent expansions:

$$
\varphi_{\nu, t+s}(\bar{x})=\bar{x}+\sum_{i \geq 1}(t+s)^{i} \nu_{i}(x)
$$

and

(4)

$$
\begin{aligned}
\varphi_{\nu, s} & \circ \varphi_{\nu, t}(\bar{x}) \\
& =\bar{x}+\sum_{i \geq 1} t^{i} \nu_{i}(\bar{x})+s \nu_{1}\left(\bar{x}+\sum_{i \geq 1} t^{i} \nu_{i}(\bar{x})\right)+\sum_{i \geq 2} s^{i} \nu_{i}\left(\bar{x}+\sum_{n \geq 1} t^{n} \nu_{n}(\bar{x})\right) .
\end{aligned}
$$

Suppose by induction that $j_{k}\left(\nu_{i}\right)\left(\overline{0}_{r}\right)=\overline{0}$ for $i \leq l$ where $l \geq 1$. Assume that $j^{k}\left(\nu_{l+1}\right)\left(\overline{0}_{r}\right) \neq \overline{0}$. By $(3)$, we obtain

$$
\begin{array}{r}
j^{k}\left(\varphi_{\nu, t+s}\right)\left(\overline{0}_{r}\right)=j_{k}\left(\operatorname{id}_{\mathbb{C}^{r}}\right)\left(\overline{0}_{r}\right)+(t+s)^{l+1} j^{k}\left(\nu_{l+1}\right)\left(\overline{0}_{r}\right) \\
+(\text { higher order terms in }(t+s)) .
\end{array}
$$


At the same time (4) implies

$$
\begin{aligned}
j^{k}\left(\varphi_{\nu, s} \circ \varphi_{\nu, t}\right)\left(\overline{0}_{r}\right)=j^{k}\left(\operatorname{id}_{\mathbb{C}^{r}}\right)\left(\overline{0}_{r}\right)+\left(t^{l+1}+s^{l+1}\right) j^{k}\left(\nu_{l+1}\right)\left(\overline{0}_{r}\right) \\
+(\text { higher order terms in } t \text { and } s) .
\end{aligned}
$$

Since $l+1 \geq 2$ this leads to contradiction.

3.2. Definition. Let $T \rightarrow N$ be a holomorphic vector bundle on a complex manifold $N, \mathcal{O}(T)$ be the sheaf of germs of holomorphic sections of $T$, and $p$ be a point of $N$. A linear subspace $V \subset \mathcal{O}_{p}(T)$ will be called $k$-sufficient if the set of $k$-jets of germs from $V$ coincides with the whole stalk $J_{p}^{k}(T)$, i.e. for any germ $\nu \in \mathcal{O}_{p}(T)$ there exists a germ $\mu \in V$ such that $j^{k}(\mu)(p)=j^{k}(\nu)(p)$. A linear subspace $W \subset H^{0}(N, \mathcal{O}(T))$ will be called $k$-sufficient at $p$ if the subspace $V$ of germs at $p$ of sections from $W$ is $k$-sufficient.

3.3. Denote by $\mathcal{T}_{s}$ the ${ }_{s} \mathcal{O}$-module of germs of holomorphic vector fields at the origin $\overline{0}_{s} \in \mathbb{C}^{s}$. If $V \subset \mathcal{T}_{s}$ is a finite dimensional subspace then all germs from $V$ can be represented by vector fields holomorphic in a common neighborhood $U \ni \overline{0}_{s}$ in $\mathbb{C}^{s}$. Moreover, one can choose $U$ so that all phase flows $\varphi_{\nu, t}$ are defined correctly on $U$ for every $\nu \in V$ and sufficiently small $|t|$. Furthermore, we may suppose that $\varphi_{\nu, t}$ is defined correctly on $U$ for every $t \in \bar{\Delta}=\{t \in \mathbb{C}|| t \mid<1\}$ as soon as $\nu \in \omega$, where $\omega$ is a neighborhood of the zero germ $\overline{0}_{V}$ in $V$.

Consider the holomorphic mapping $\rho_{V}: \omega \ni \nu \longmapsto \varphi_{\nu, 1} \in \operatorname{Hol}\left(U, \mathbb{C}^{s}\right)$ and its $k$-jet extension at the origin $\rho_{V, k}: \omega \ni \nu \longmapsto j^{k}\left(\varphi_{\nu, 1}\right)\left(\overline{0}_{s}\right) \in J_{\overline{0}_{s}}^{k}\left(\left(\mathbb{C}^{s}, \overline{0}_{s}\right), \mathbb{C}^{s}\right)$.

3.4. Lemma. Let a finite dimensional subspace $V \subset \mathcal{T}_{s}$ be $k$-sufficient. Then the differential $d \rho_{V, k}$ at the origin $\overline{0}_{V} \in \omega \subset V$ is surjective.

Proof. Since $\varphi_{t_{1} \nu, t_{2}}=\varphi_{\nu, t_{1} t_{2}}$ we have $\varphi_{t \nu, 1}=\varphi_{\nu, t}$. Applying expansions (1) and (2) one obtains that for $\nu \in V$

$$
\rho_{V, k}(t \nu)=j^{k}\left(\operatorname{id}_{\mathbb{C}^{s}}\right)\left(\overline{0}_{s}\right)+t j^{k}(\nu)\left(\overline{0}_{s}\right)+\sum_{i \geq 2} t^{i} j^{k}\left(\nu_{i}\right)\left(\overline{0}_{s}\right) .
$$

Therefore, the derivative $\partial \rho_{V, k} / \partial \nu$ at the direction $\nu$ at the origin $\overline{0}_{V} \in V$ is equal to $j^{k}(\nu)\left(\overline{0}_{s}\right)$. Since $V$ is $k$-sufficient the image of the differential $d \rho_{V, k}\left(\overline{0}_{V}\right)$ coincides with the whole stalk $J_{\overline{0}_{s}}^{k}\left(T \mathbb{C}^{s}\right)=J_{\overline{0}_{s}}^{k}\left(\left(\mathbb{C}^{s}, \overline{0}_{s}\right), \mathbb{C}^{s}\right)$.

3.5. For $r \leq s$ we regard $\mathbb{C}^{r}$ as a coordinate subspace in $\mathbb{C}^{s}: \mathbb{C}^{r}=\left\{\bar{x}^{\prime} \in \mathbb{C}^{s} \mid \bar{x}^{\prime}=\right.$ $\left.\left(x_{1}, \ldots, x_{r}, 0, \ldots, 0\right)\right\}$. The restriction to this subspace defines for any nonnegative integer $k$ the projection $\pi_{s, r}^{k}: J^{k}\left(\left(\mathbb{C}^{s}, \overline{0}_{s}\right), \mathbb{C}^{s}\right) \rightarrow J^{k}\left(\left(\mathbb{C}^{r}, \overline{0}_{r}\right), \mathbb{C}^{s}\right)$.

Let $V$ and $\omega$ be as in 3.3. Consider the holomorphic mapping

$$
\Phi=\Phi_{V, r, k}: \omega \times\left(\mathbb{C}^{r}, \overline{0}_{r}\right) \rightarrow J^{k}\left(\left(\mathbb{C}^{r}, \overline{0}_{r}\right), \mathbb{C}^{s}\right)
$$

given by the formula

$$
\Phi\left(\nu, \bar{x}^{\prime}\right)=\pi_{s, r}^{k} \circ j^{k}\left(\varphi_{\nu, 1}\right)\left(\bar{x}^{\prime}\right) .
$$

We have the following commutative diagram: 


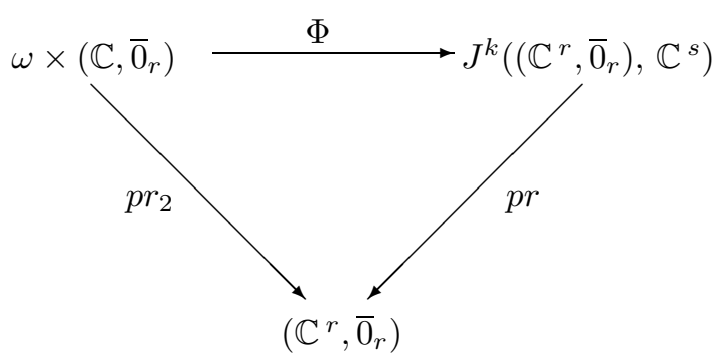

where $p r_{2}$ and $p r$ are the canonical projections. Since the restriction

$$
\left.\Phi\right|_{\left(p r_{2}\right)^{-1}\left(\overline{0}_{r}\right)}: \omega \rightarrow J_{\overline{0}_{r}}^{k}\left(\left(\mathbb{C}^{r}, \overline{0}_{r}\right), \mathbb{C}^{s}\right)=p r^{-1}\left(\overline{0}_{r}\right)
$$

coincides with the composition $\pi_{s, r}^{k} \circ \rho_{V, k}$, the above diagram and Lemma 3.4 imply the following fact.

3.6. Corollary. Let a finite dimensional subspace $V \subset \mathcal{T}_{s}$ be $k$-sufficient. Then the differential $d \Phi$ at the origin $\overline{0}_{V} \times \overline{0}_{r} \in V \times \mathbb{C}^{r}$ is surjective.

3.7. Lemma. Let $Y$ be a closed analytic subset of a Stein manifold $X$ and let $x_{0} \in X \backslash Y$. Suppose that $T$ is a holomorphic vector bundle on $X$. Then for every nonnegative integer $k$ there exists a holomorphic section $\sigma$ of $T$ with a given $k$-jet $\nu$ at $x_{0}$ and such that $\left.j^{k}(\sigma)\right|_{Y} \equiv \overline{0}$.

Proof. Let $I_{Y}$ (resp. $I_{x_{0}}$ ) be the ideal sheaf of $Y$ (resp. $\left.x_{0}\right)$ in $\mathcal{O}_{X}$. Put

$$
\mathcal{T}=\mathcal{O}_{X}(T), \quad \mathcal{T}^{\prime}=\mathcal{T} \otimes_{\mathcal{O}_{X}}\left(I_{Y}\right)^{k}, \quad \mathcal{T}^{\prime \prime}=\mathcal{T}^{\prime} \otimes_{\mathcal{O}_{X}}\left(I_{x_{0}}\right)^{k+1} .
$$

Note that $\mathcal{T}^{\prime}$ (resp. $\mathcal{T}^{\prime \prime}$ ) can be regarded as a subset of $\mathcal{T}$ (resp. $\mathcal{T}^{\prime}$ ). Put $J=$ $\mathcal{T}^{\prime} / \mathcal{T}^{\prime \prime}$. Then $\left.J\right|_{X \backslash\left\{x_{0}\right\}}$ is trivial. It is also clear that $\left.J\right|_{\left\{x_{0}\right\}}$ coincides with the stalk $J_{x_{0}}^{k}(T)$ of $k$-jets of germs at $x_{0}$ of holomorphic sections of $T$. Thus every $k$-jet $\nu$ at $x_{0}$ may be viewed as a global holomorphic section $\sigma_{0}$ of $J$. By the Cartan Theorem $\mathrm{B}$ applied to the coherent sheaf $\mathcal{T}^{\prime \prime}$, we have $H^{1}\left(X, \mathcal{T}^{\prime \prime}\right)=0$. Hence the exact cohomology sequence implies that the section $\sigma_{0}$ is the image of a holomorphic section $\sigma \in H^{0}\left(X, \mathcal{T}^{\prime}\right)$. Clearly, $\sigma$ is a section of $T$ with the desired properties.

3.8. Corollary. Under the assumption of Lemma 3.7 there exists a finite dimensional subspace $V \subset H^{0}(X, \mathcal{O}(T))$ such that $\left.j^{k}(\sigma)\right|_{Y} \equiv \overline{0}$ for all $\sigma \in V$ and $V$ is $k$-sufficient at $x_{0}$, i.e. $J_{x_{0}}^{k}(T)=J_{x_{0}}^{k}(V)$.

Proof. Choose a basis $v_{1}, \ldots, v_{N}$ of $J_{x_{0}}^{k}(T)$. By Lemma 3.7, for each $i=1, \ldots, N$ there exists $\nu_{i} \in H^{0}(X, \mathcal{O}(T))$ such that $\left.j^{k}\left(\nu_{i}\right)\right|_{Y} \equiv 0$ and $j^{k}\left(\nu_{i}\right)\left(x_{0}\right)=v_{i}$. Put $V=\operatorname{span}\left(\nu_{1}, \ldots, \nu_{N}\right)$.

Denote by $\mathcal{T}_{M}=H^{0}(M, \mathcal{O}(T M))$ the space of global holomorphic vector fields on a complex manifold $M$.

3.9. Lemma. Let $X$ be a closed submanifold of a Stein manifold $M, Y$ be a closed analytic subset of $X$, and $K \subset X, K^{\prime} \subset K \backslash Y$ be compact subsets. Then there exist a neighborhood $U$ of $K$ in $X$ (resp. $U^{\prime}$ of $K^{\prime}$ in $X \backslash Y$ ), a finite dimensional subspace $V \subset \mathcal{T}_{M}$, and a neighborhood $\omega$ of the origin $\overline{0}_{V} \in V$ such that the following conditions hold:

(i) $\left.j^{k}(v)\right|_{Y} \equiv \overline{0}$ for each $\nu \in V$; 
(ii) for every $\nu \in \omega$ the associated phase flow $\varphi_{\nu, t}$ is defined on $U \times \bar{\Delta}$;

(iii) if $\Phi$ is the holomorphic mapping $\Phi: \omega \times U \ni(\nu, x) \longmapsto j^{k}\left(\varphi_{\nu, 1}\right)(x) \in$ $J^{k}(U, M)$ then the differential $d \Phi$ is surjective at each point $\left(\nu, x^{\prime}\right) \in \omega \times U^{\prime}$.

Proof. By Corollary 3.8, for each point $x^{\prime} \in K^{\prime}$ there exists a finite dimensional subspace $V_{x^{\prime}} \subset \mathcal{J}_{M}$ which is $k$-sufficient at $x^{\prime}$ and satisfies the above condition (i). Of course, $V_{x^{\prime}}$ is $k$-sufficient at each point of some small neighborhood $U_{x^{\prime}}^{\prime}$ of $x^{\prime}$ in $X$. Let $\left\{U_{x_{i}^{\prime}}^{\prime}\right\}_{i=1}^{N^{\prime}}$ be a finite covering of $K^{\prime}$ by such neighborhoods. Put $U^{\prime}=\bigcup_{i=1}^{N^{\prime}} U_{x_{i}^{\prime}}^{\prime}$ and $V=\bigoplus_{i=1}^{N^{\prime}} V_{x_{i}^{\prime}}$. Then $V$ is a finite dimensional subspace of $\mathcal{T}_{M}$ which satisfies (i) and is $k$-sufficient at each point $x^{\prime} \in U^{\prime}$.

Furthermore, for each point $x \in K$ there exist a neighborhood $U_{x}$ in $X$ and a neighborhood $\omega_{x} \ni \overline{0}_{V}$ in $V$ such that for every $\nu \in \omega_{x}$ the associated phase flow $\varphi_{\nu, t}$ is holomorphic in $U_{x} \times \bar{\Delta}$. Choose a finite covering $\left\{U_{x_{j}}\right\}_{j=1}^{N}$ of the compact $K$ in $X$ and put $U=\bigcup_{j=1}^{N} U_{x_{j}}, \omega=\bigcap_{j=1}^{N} \omega_{x_{j}}$. Then $U$ and $\omega$ satisfy condition (ii). By Corollary 3.6, condition (iii) also holds.

Next we give an analytic version of the Collective Transversality Lemma [AVG].

3.10. Lemma. Under the assumption of Lemma 3.9 for every stratified analytic subset $A \subset J^{k}(X, M)$ there exists a dense subset $\omega_{0}$ of $\omega$ such that for each $\nu \in \omega_{0}$ the mapping

$$
\left.j^{k}\left(\varphi_{\nu, 1}\right)\right|_{U^{\prime}}: U^{\prime} \rightarrow J^{k}\left(U^{\prime}, M\right) \subset J^{k}(X, M)
$$

is transversal to $A$.

Proof. Denote by $\left(A_{\sigma}\right)_{\sigma \in \Sigma}$ the collection of strata of the given stratification $\Sigma$ of $A$ which satisfies Whitney's condition (a). Put $\tilde{A}=\Phi^{-1}(A) \subset \omega \times U^{\prime}$. Since $d \Phi$ is surjective at each point $\left(\nu, x^{\prime}\right) \in \omega \times U^{\prime}$ it is clear that $\left\{\tilde{A}_{\sigma}=\Phi^{-1}\left(A_{\sigma}\right)\right\}_{\sigma \in \Sigma}$ is an analytic stratification of $\tilde{A}$ which also satisfies Whitney's condition (a). Furthermore, the mapping $\left.j^{k}\left(\varphi_{\nu, 1}\right)\right|_{U^{\prime}}: U^{\prime} \rightarrow J^{k}(X, M)$ is transversal to $A$ iff the identical embedding $U_{\nu}^{\prime}:=\{\nu\} \times U^{\prime} \hookrightarrow \omega \times U^{\prime}$ is transversal to $\tilde{A}$. The latter means that for each $\sigma \in \Sigma$ the differential of the canonical projection $p r_{1}: \omega \times U^{\prime} \rightarrow \omega$ restricted to $\tilde{A}$ is surjective at each of the sets $U_{\nu}^{\prime} \cap \tilde{A}_{\sigma}$. Put $\pi_{\sigma}=\left.p r_{1}\right|_{\tilde{A}_{\sigma}}: \tilde{A}_{\sigma} \rightarrow \omega$ and $S_{\sigma}=\left\{x \in \tilde{A}_{\sigma} \mid \operatorname{rank} d \pi_{\sigma}(x)<\operatorname{dim} \omega\right\}$. By Proposition I.3.8 in [Ch], the image $\pi_{\sigma}\left(S_{\sigma}\right) \subset \omega$ is contained in at most countable union of proper analytic subsets of $\omega$ (not necessarily closed). The same is valid for the "discriminant" set $D=\bigcup_{\sigma \in \Sigma} \pi_{\sigma}\left(S_{\sigma}\right) \subset \omega$. Therefore, the desired transversality condition holds for any $\nu$ in the dense subset $\omega_{0}=\omega \backslash D$ of $\omega$.

\section{Proof of the Transversality Theorem}

4.1. Lemma. Let $X$ be a closed submanifold of a Stein manifold $M, Y$ be a closed analytic subset of $X$, and $K$ be a compact in $X$. Suppose that $A$ is a stratified analytic subset of $J^{k}(X, M)$. Let $f$ be the identical embedding and let $\left.j^{k}(f)\right|_{Y} \pitchfork A$. Then in a neighborhood $\Omega$ of $K$ in $X$ the mapping $f$ can be uniformly approximated 
(with respect to a given Hermitian metric $h$ on $M$ ) by holomorphic mappings $\tilde{f}$ : $\Omega \rightarrow M$ such that $j^{k}(\tilde{f}) \pitchfork A$ and $\left.j^{k}(\tilde{f})\right|_{Y \cap \Omega}=\left.j^{k}(f)\right|_{Y \cap \Omega}$.

Proof. Since the given stratification $\Sigma$ of $A$ satisfies Whitney's condition (a) and $\left.j^{k}(f)\right|_{Y} \pitchfork A$, there exists a neighborhood $W$ of $Y$ in $X$ for which $\left.j^{k}(f)\right|_{W} \pitchfork A$. Put $K^{\prime}=K \backslash W^{\prime}$, where $W^{\prime} \subset W$ is a smaller neighborhood of $Y$ such that $\bar{W}^{\prime} \subset W$. Let $U \supset K, U^{\prime} \supset K^{\prime}, V \subset \mathcal{T}_{M}$, and $\omega \subset V$ be the same as in Lemma 3.9. Let $\Omega$ be a neighborhood of $K$ so that $\Omega \subset \subset\left(U^{\prime} \cup W^{\prime}\right) \cap U$. Put $f_{\nu}=\left.\varphi_{\nu, 1}\right|_{\Omega}$ for $\nu \in \omega$. Since $(A, \Sigma)$ satisfies Whitney's condition (a) and $\left.j^{k}\left(f_{\nu}\right) \rightarrow j^{k}(f)\right|_{\Omega}$ as $\nu \rightarrow \overline{0}_{V} \in V$ there exists a smaller neighborhood $\omega^{\prime} \subset \omega$ of the origin $\overline{0}_{V} \in V$ for which $\left.j^{k}\left(f_{\nu}\right)\right|_{W^{\prime} \cap \Omega} \pitchfork A$ for all $\nu \in \omega^{\prime}$. By Lemma 3.10, we also have $\left.j_{k}\left(f_{\nu}\right)\right|_{U^{\prime} \cap \Omega} \pitchfork A$ for every $\nu$ in a dense subset $\omega_{0}^{\prime}$ of $\omega^{\prime}$. So, $\left.j^{k}\left(f_{\nu}\right)\right|_{\Omega} \pitchfork A$ for every $\nu \in \omega_{0}^{\prime}$. Condition $\left.j^{k}(\nu)\right|_{Y} \equiv \overline{0}$ and Lemma 3.1 imply that $\left.j^{k}\left(f_{\nu}\right)\right|_{Y \cap \Omega}=$ $\left.j^{k}\left(\varphi_{\nu, 1}\right)\right|_{Y \cap \Omega}=\left.j^{k}\left(\operatorname{id}_{X}\right)\right|_{Y \cap \Omega}=\left.j^{k}(f)\right|_{Y \cap \Omega}$. We conclude the proof by noting that $\tilde{f}=f_{\nu}$ uniformly converges to $\left.f\right|_{\Omega}=$ id on $\Omega$ when $\nu \in \omega_{0}^{\prime}$ approaches $\overline{0}_{V}$.

The rest of this section is a reduction of Theorem 1.2 to Lemma 4.1. The first step is to replace $f=\mathrm{id}_{X}$ in Lemma 4.1 by an arbitrary holomorphic mapping $f: X \rightarrow M$.

4.2. Lemma. Let $X$ be a Stein manifold, $M$ be a complex manifold endowed by a Hermitian metric $h, f: X \rightarrow M$ be a holomorphic mapping. Let $Y, K, A$ be the same as in Lemma 4.1, and as before $j^{k}(f) \pitchfork A$. Then the conclusion of Lemma 4.1 holds for such $f$.

Proof. Let $F=\left(\operatorname{id}_{X}, f\right)$ be the embedding of $X \hookrightarrow X \times M$ onto the graph of $f$. In sequel we identify $X$ with its image under $F$ and consider $F$ as the identical embedding. Denote by $p r_{*}: j^{k}(X, X \times M)=J^{k}(X, X) \times J^{k}(X, M) \rightarrow$ $J^{k}(X, M)$ the forgetting projection. It is easily seen that the analytic subset $\tilde{A}=\left(p r_{*}\right)^{-1}(A) \subset J^{k}(X, X \times M)$ with the induced stratification is a stratified analytic subset, i.e. the Whitney condition (a) holds. Furthermore, the assumption $\left.j_{k}(f)\right|_{Y} \pitchfork A$ yields $\left.j^{k}(F)\right|_{Y} \pitchfork \tilde{A}$. Let $g$ be any Hermitian metric on $X$ and let $\tilde{h}$ be the Hermitian metric on $X \times M$ that is the Euclidian sum of $g$ and $h$. By Siu's theorem [S], the Stein submanifold $X=F(X) \hookrightarrow X \times M$ admits a Stein neighborhood $M^{\prime}$ in $X \times M$. By Lemma 4.1 applied to the identical embedding $F$ of $X=F(X)$ into the Stein manifold $M^{\prime}$, in a neighborhood $\Omega$ of a compact $K$ the mapping $F$ can be uniformly approximated (with respect to the metric $\tilde{h}$ ) by holomorphic mappings $\tilde{F}: \Omega \rightarrow M^{\prime} \hookrightarrow X \times M$ such that $\left.j^{k}(\tilde{F})\right|_{Y \cap \Omega}=\left.j^{k}(F)\right|_{Y \cap \Omega}$ and $j^{k}(\tilde{F}) \pitchfork \tilde{A}$. Put $\tilde{f}=p r_{M} \circ \tilde{F}$ where $p r_{M}: X \times M \rightarrow M$ is the canonical projection. Clearly, $\tilde{f}$ uniformly approximates $\left.f\right|_{\Omega}$ with respect to the metric $h$ on $M,\left.j^{k}(\tilde{f})\right|_{Y \cap \Omega}=\left.j^{k}(f)\right|_{Y \cap \Omega}$, and $j^{k}(\tilde{f}) \pitchfork A$. Therefore, all conclusions of Lemma 4.1 hold.

4.3. Proof of Theorem 1.2. For any holomorphic submersion $\varphi: E \rightarrow B$ of complex manifolds which is a smooth fiber bundle and for every Hermitian metric $h$ on $B$, using smooth partition of unity, one can construct a Hermitian metric $\tilde{h}$ on $E$ so that $\varphi^{*} h \leq \tilde{h}$. By this simple remark, we may fix a sequence of Hermitian metrics 
$h_{l}$ on $J^{l}(X, M)$ such that all natural projections $\pi_{m, n}: J^{m}(X, M) \rightarrow J^{n}(X, M)$ (where $n<m$ ) and $\pi_{l}: J^{l}(X, M) \rightarrow M$ ( $M$ being endowed with a Hermitian metric $h)$ are contractions with respect to these metrics. Let $U \subset X$ be an open subset and $g: U \rightarrow M$ be a holomorphic mapping. If $A \subset J^{n}(X, M)$ is a stratified analytic subset and for $m>n$ the preimage $\pi_{m, n}^{-1}(A)$ is endowed with the induced stratification, then it is easily seen that conditions $j^{n}(g) \pitchfork A$ and $j^{m}(g) \pitchfork \pi_{m, n}^{-1}(A)$ are equivalent. Hence, passing to appropriate preimages of the given stratified analytic subsets $A_{i} \subset J^{k_{i}}(X, M), i=1, \ldots$, we may suppose that $k \leq k_{i}<k_{j}$ for all $i<j, i, j=1, \ldots$. Next we fix a relatively compact neighborhood $\Omega$ of $K$ in $X$ which is a Runge domain in $X$. To see that $\Omega$ exists it is enough to take the intersection of the Stein manifold $X$ embedded in $\mathbb{C}^{N}$ with a large ball $B_{R}^{N}$ containing the image of $K$. Fix also a bigger Runge neighborhood $\Omega_{0}$ (for instance, put $\left.\Omega_{0}=X \cap B_{R+1}^{N}\right)$. Replacing $K$ by the compact $L=\bar{\Omega}_{0}$, we will construct by induction a decreasing sequence $\ldots \subset \subset \Omega_{l+1} \subset \subset \Omega_{l} \subset \subset \cdots \subset \subset \Omega_{1}$ of neighborhoods of $L$ in $X$ and a sequence of holomorphic mappings $f_{l}: \Omega_{l} \rightarrow M$ which satisfy

(1 $1_{l} j^{k_{l}}\left(f_{l}\right) \pitchfork A_{l}$,

(2) $\left.j^{k}\left(f_{l}\right)\right|_{Y \cap \Omega_{l}}=\left.j^{k}(f)\right|_{Y \cap \Omega_{l}}$,

$\left(3_{l}\right) \operatorname{dist}_{h_{k_{l}}}\left(j^{k_{l}}\left(f_{l}\right), j^{k_{l}}\left(f_{l-1}\right)\right)<\delta_{l} \leq 2^{-l} \varepsilon$ uniformly on $\Omega_{l}$,

where $\delta_{l}>0$ will be defined later on, and where for $l=1$ in $\left(3_{l}\right) f_{0}:=f$. By our choice of Hermitian metrics $h_{i}$, given such a sequence $\left\{f_{l}\right\}$, we have

$\left(3^{\prime}\right) \operatorname{dist}_{h}\left(f_{l}, f_{l-1}\right)<2^{-l} \varepsilon$

for all $l$ uniformly in $\Omega_{0}$. Therefore, $f_{l} \stackrel{l}{\longrightarrow} \tilde{f}$ uniformly in $\Omega_{0}$ where $\tilde{f} \in \operatorname{Hol}\left(\Omega_{0}, M\right)$ satisfies condition (2) and (3) of Theorem 1.2. To ensure condition (1) we need to choose $\left\{\delta_{l}\right\}$ in an appropriate way.

By the Cauchy integral formula, given an open subset $U \subset X$ and a sequence $g_{i} \in \operatorname{Hol}(U, M)$ convergent to $g \in \operatorname{Hol}(U, M)$ uniformly on a compact subset $R \subset$ $U$, we have a uniform convergence on $R$ of the $l$-jet extensions $j^{l}\left(g_{i}\right)$ to $j^{l}(g)$ for every natural $l$. Thus, by Lemma 4.2 , there exists a neighborhood $\Omega_{1}$ of $L$ in $X$ and a holomorphic mapping $f_{1}: \Omega_{1} \rightarrow M$ such that $j^{k_{1}}\left(f_{1}\right) \pitchfork A_{1},\left.j^{k_{1}}\left(f_{1}\right)\right|_{Y \cap \Omega_{1}}=$ $\left.j^{k_{1}}(f)\right|_{Y \cap \Omega_{1}}$, and $\operatorname{dist}_{h_{k_{1}}}\left(j^{k_{1}}\left(f_{1}\right), j^{k_{1}}(f)\right)<\varepsilon / 2$. Hence $f_{1}$ satisfies $\left(1_{1}\right)-\left(3_{1}\right)$ with $\delta_{1}=\varepsilon / 2$. Passing to a smaller neighborhood of $L$ we may suppose that $\Omega_{1}$ is Stein. Let $\delta_{i}, i=1, \ldots, l$, be already constructed so that $f_{i}$ satisfies $\left(1_{i}\right)-\left(3_{i}\right)$ for $i \leq l$. By Lemma 4.2 and the note above applied to the holomorphic mapping $f_{l}: \Omega_{l} \rightarrow M$, for every $\delta_{l+1}$ such that $0<\delta_{l+1}<2^{-(l+1)} \varepsilon$ there exist a smaller Stein neighborhood $\Omega_{l+1} \subset \subset \Omega_{l}$ of the compact $L$ in $X$ and a holomorphic mapping $f_{l+1}: \Omega_{l+1} \rightarrow M$ which satisfy $\left(1_{l+1}\right)-\left(3_{l+1}\right)$. Now for each $i=1,2, \ldots, l$ we have $\operatorname{dist}_{h_{k_{i+1}}}\left(\left.j^{k_{i+1}}\left(f_{i}\right)\right|_{\Omega_{l+1}}, j^{k_{i+1}}\left(f_{l+1}\right)\right)<\sum_{p=i+1}^{l+1} \delta_{p}$. Since $k_{i}<k_{i+1}$ and, by condition (1), $j^{k_{i}}\left(f_{i}\right) \pitchfork A_{i}$ there exists $\mu_{i}>0$ such that for any $g \in \operatorname{Hol}\left(\Omega_{0}, M\right)$ the condition

$$
\operatorname{dist}_{h_{k_{i+1}}}\left(j^{k_{i+1}}(g), j^{k_{i+1}}\left(f_{i}\right)\right)<\mu_{i}
$$

in $\Omega_{0}$ yields $\left.j^{k_{i}}(g)\right|_{\Omega} \pitchfork A_{i}$. So, we will choose $\left\{\delta_{i}\right\}$ in such a way that

$$
\sum_{p=i+1}^{\infty} \delta_{p}<\mu_{i}
$$


for every $i=1, \ldots$. By induction, we may suppose that the condition

$$
\rho_{i}:=\sum_{p=i+1}^{l} \delta_{p}<\mu_{i} / 2, \quad i=1, \ldots, l-1,
$$

is already true. Choose $\delta_{l+1}, 0<\delta_{l+1}<2^{-(l+1)} \varepsilon$ so that $\delta_{l+1}<\min _{1 \leq i \leq l-1}\left(\mu_{i} / 2-\rho_{i}\right)$ and $\delta_{l+1}<\mu_{l} / 2$. This implies (6) for every $i=1, \ldots$ Therefore, the limit mapping $g:=\tilde{f}=\lim _{l \rightarrow \infty} f_{l} \in \operatorname{Hol}\left(\Omega_{0}, M\right)$ satisfies (5). Hence $\left.j^{k_{i}}(\tilde{f})\right|_{\Omega} \pitchfork A_{i}$ which completes the proof of Theorem 1.2.

\section{Stability OF EISEnMan InTRINSic $k$-MEASURES}

5.1. Proof of Corollary 1.5. Denote by $\bar{e}_{k}$ the $k$-vector

$$
\frac{\partial}{\partial z_{1}} \wedge \cdots \wedge \frac{\partial}{\partial z_{k}} \in \Lambda^{k} T_{\overline{0}_{k}} B^{k}
$$

at the origin $\overline{0}_{k} \in B^{k} \subset \mathbb{C}^{k}$. Recall $[\mathrm{E}],[\mathrm{P}],[\mathrm{Ko} 1],[\mathrm{GW}]$ that for a complex manifold $M$ and a decomposable vector $\bar{v}=v_{1} \wedge \cdots \wedge v_{k} \in \Lambda^{k} T_{p} M$, where $p \in M$ and $1 \leq k \leq m=\operatorname{dim} M$, the Eisenman $k$-measure of $\bar{v}$ is defined by the formula

$$
\begin{aligned}
& E_{M}^{k}(p, \bar{v})=\inf \left\{\lambda^{2} \mid \lambda>0 \text { is such that there exists } f \in \operatorname{Hol}\left(B^{k}, M\right)\right. \\
& \text { with } \left.f\left(\overline{0}_{k}\right)=p \text { and } d f\left(\lambda \bar{e}_{k}\right)=\bar{v}\right\} .
\end{aligned}
$$

If $A$ is a closed analytic subset of $M$ of codimension at least $k+1$ and $p \in M \backslash A$ then, by Transversality Theorem 1.2, every holomorphic mapping $f: B^{k} \rightarrow M$ with $f\left(\overline{0}_{k}\right)=p$ and $d f\left(\lambda \bar{e}_{k}\right)=\bar{v}$ can be approximated by holomorphic mappings of smaller balls $f_{\varepsilon}: B_{1-\varepsilon}^{k} \rightarrow M \backslash A$ with $f_{\varepsilon}\left(\overline{0}_{k}\right)=p$ and $d f_{\varepsilon}\left(\lambda e_{k}\right)=v$. This observation implies Corollary 1.5 in the Introduction.

Indeed, consider the mapping $\tilde{f}_{\varepsilon}(z)=f_{\varepsilon}((1-\varepsilon) z), \tilde{f}_{\varepsilon} \in \operatorname{Hol}\left(B^{k}, M \backslash A\right), \tilde{f}_{\varepsilon}\left(\overline{0}_{k}\right)$ $=p, d \tilde{f}_{\varepsilon}\left(\bar{e}_{k}\right)=(1-\varepsilon) \lambda \bar{v}$. By the above definition, we obtain

$$
E_{M \backslash A}^{k}(p, \bar{v}) \leq E_{M}^{k}(p, \bar{v}) .
$$

The opposite inequality holds since embeddings do not increase Eisenman measures.

In [PS] it was mentioned that the Campbell-Howard-Ochiai-Ogawa Stability Theorem is still true for any subset of $M$ of Hausdorff $(2 m-2)$-measure zero. Here we present the similar generalization of Corollary 1.5.

5.2. Proposition. Let $M$ be a connected complex manifold of complex dimension $m$ and let $A$ be its subset of Hausdorff $(2 m-2 k)$-measure 0 for some $k \in$ $\{1,2, \ldots, m\}$. Then $E_{M \backslash A}^{k}=E_{M}^{k} \mid M \backslash A$.

The proof is similar to the proof of Corollary 1.5, but instead of Theorem 4.3 we have to use the following modification of it.

5.3. Theorem. Let $M$ be a connected complex manifold of $\operatorname{dim}_{\mathbb{C}} M=m$ with a Hermitian metric $h$, and let $A$ be its closed subset of Hausdorff $(2 m-2 k)$-measure zero for some $k \in\{1, \ldots, m\}$. Let $X$ be a Stein manifold of $\operatorname{dim}_{\mathbb{C}} X=k, Y$ be a closed analytic subset of $X$, and $f: X \rightarrow M$ be a holomorphic mapping such that $f(Y) \subset M \backslash A$. Then for any compact $K \subset X$ the mapping $f$ can be uniformly (with respect to $h$ ) approximated in a neighborhood $\Omega$ of $K$ by holomorphic mappings $\tilde{f}: \Omega \rightarrow M \backslash A$ such that $\left.j^{1}(f)\right|_{Y \cap \Omega}=\left.j^{1}(\tilde{f})\right|_{Y \cap \Omega}$. 
Proof. We follow the lines of the proof of Theorem 1.2. As in Lemma 4.2, replacing $f$ by the embedding $F=\left(\operatorname{id}_{X}, f\right)$ onto the graph of $f$ and replacing $M$ by a Stein neighborhood of the graph in $X \times M$, we may suppose that $M$ is Stein, $X$ is a closed submanifold of $M$, and $f$ is the identical embedding $X \hookrightarrow M$. Note that under this replacement the codimension of $X$ in $M$ is still equal to the dimension of vanishing Hausdorff measure of $A$ ( $A$ being replaced by $\operatorname{pr}_{2}^{-1}(A) \subset X \times M$ ). Since $f(Y) \cap A=\emptyset$ there exists a neighborhood $W$ of $Y \cap K$ in $X$ such that $f(W) \cap A=W \cap A=\emptyset$. Fix a smaller relatively compact neighborhood $W^{\prime} \subset \subset W$ and $\varepsilon>0$ such that for each $\tilde{f} \in \operatorname{Hol}\left(W^{\prime}, M\right)$ with $\operatorname{dist}_{h}\left(\tilde{f},\left.f\right|_{W^{\prime}}\right)<\varepsilon$ uniformly in $W^{\prime}$ one has $\tilde{f}\left(W^{\prime}\right) \cap A=\emptyset$. Put $K^{\prime}=K-W^{\prime}$. By Lemma 3.9, there exist neighborhoods $U$ of $K$ in $X, U^{\prime}$ of $K^{\prime}$ in $X$, a finite dimensional subspace $V \subset \mathcal{T}_{M}$, and a neighborhood $\omega^{\prime}$ of the origin $\overline{0}_{V} \in V$ such that the following conditions hold:

(i) $\left.j^{1}(\nu)\right|_{Y} \equiv \overline{0}$ for every $\nu \in V$;

(ii) $\Phi \in \operatorname{Hol}\left(\omega^{\prime} \times U, M\right)$ where $\Phi(\nu, z)=\varphi_{\nu, 1}(z)$;

(iii) $\operatorname{dist}_{h}\left(\left.\varphi_{\nu, 1}\right|_{U},\left.f\right|_{U}\right)<\varepsilon$ uniformly in $U$;

(iv) the rank of $d \Phi$ is $m=\operatorname{dim}_{\mathbb{C}} M$ at each point $z \in U^{\prime}$.

By Lemma 3.1, (i) and (ii) imply that $\left.\varphi_{\nu, 1}\right|_{U \cap Y}=\left.f\right|_{U \cap Y}=$ id $\left.\right|_{U \cap Y}$, and, by (iii), $\varphi_{\nu, 1}\left(U \cap W^{\prime}\right) \subset M \backslash A$ for each $\nu \in \omega^{\prime}$. By virtue of Theorem 2 from [Ch, Appendix, 2] condition (iv) implies that the mapping $\left.\Phi\right|_{\omega^{\prime} \times U^{\prime}}$ may be locally treated as a projection. From Proposition 7 in [Ch, Appendix, 6] it easily follows that the preimage $A^{\prime}=\left(\left.\Phi\right|_{\omega^{\prime} \times U^{\prime}}\right)^{-1}(A) \subset \omega^{\prime} \times U^{\prime}$ has Hausdorff $2 l$-measure zero where $l=\operatorname{dim}_{\mathbb{C}} V$. Therefore, by Property 4 of Hausdorff measures as it was listed in [Ch, Appendix, 6], we have that the image $A^{\prime \prime}$ of $A^{\prime}$ under the projection $\omega^{\prime} \times U^{\prime} \rightarrow \omega^{\prime}$ also has Hausdorff $2 l$-measure zero. It follows that $\omega^{\prime} \backslash A^{\prime \prime}$ is a dense subset of $\omega^{\prime}$. For each $\nu \in \omega^{\prime} \backslash A^{\prime \prime}$ the image $\varphi_{\nu, 1}\left(U^{\prime}\right)=\Phi\left\{\{\nu\} \times U^{\prime}\right\} \subset M$ does not meet $A$. Thus for this $\nu$ we have $\varphi_{\nu, 1}\left(U^{\prime} \cup W^{\prime}\right) \cap A=\emptyset$. Take a neighborhood $\Omega$ of $K$ in $X$ such that $\Omega \subset\left(U^{\prime} \cup W^{\prime}\right) \cap U$. Then $\tilde{f}:=\left.\varphi_{\nu, 1}\right|_{\Omega} \in \operatorname{Hol}(\Omega, M)$, where $\nu \in \omega^{\prime} \backslash A^{\prime \prime}$ and $\nu \rightarrow \overline{0}_{V}$, gives the desired approximation of $f$.

\section{REFERENCES}

[AVG] V.I. Arnold, A.N. Varchenko, S.M. Gusein-Zade, Singularities of differentiable maps, Boston, Birkhäuser, 1985. MR 86f:58018

[BG] R. Brody, M. Green, On a family of hyperbolic surfaces in $\mathbb{P}^{3}$, Duke Math. J., 44(1977), no.4, 873-874. MR 56:12331

[CHO] L. Campbell, A. Howard, T. Ochiai, Moving discs off analytic subsets, Proc. AMS, 60(1976), no.1, 106-107. MR 54:13143

[CO] L. Campbell, R. Ogawa, On preserving the Kobayashi pseudometric, Nagoya Math. J., 57(1975), no.1, 37-47. MR 51:8474

[Ch] E.M. Chirka, Complex Analytic Sets, Kluwer Academic Publish., Dordrecht, 1989. MR 92b:32016

[DLS] J.-P. Demailly, L. Lempert, B. Shiffman, Algebraic approximation of holomorphic maps from Stein domains to projective manifolds, preprint, 1993.

[E] D.A. Eisenman, Intrinsic measures on complex manifolds and holomorphic mappings, Mem. AMS, no.96, AMS, Providence, R.I., 1970. MR 41:3807

[G] M.L. Green, Some Picard theorems for holomorphic maps to algebraic varieties, Amer. J. Math., 97(1975), 43-75. MR 51:3544

[GW] I. Graham, H. Wu, Some remarks on the intrinsic measures of Eisenman, Trans. A.M.S., 288(1985), 625-660. MR 86e:32031

[Ka 1] Sh. Kaliman, Some facts about Eisenman intrinsic measures, Complex Variables, (to appear).

[Ka 2] Sh. Kaliman, Exotic analytic structures and Eisenman intrinsic measures, Israel J. Math, 88 (1994), 411-423. CMP95:04. 
[Ko 1] Sh. Kobayashi, Hyperbolic manifolds and holomorphic mappings, Pure and Appl. Math., 2, Dekker, New York, 1970. MR 43:3503

[Ko 2] Sh. Kobayashi, Intrinsic distances, measures and geometric function theory, Bull. AMS, 82(1976), 357-416. MR 54:3032

[LZ] V. Ya. Lin, M.G. Zaidenberg, Finiteness theorems for holomorphic mappings, Encyclopaedia of Math. Sci., 9(1986), 127-194 (in Russian). English transl. in Encyclopaedia of Math. Sci., Vol.9. Several Complex Variables III. Berlin-Heidelberg-New York, Springer Verlag, 1989, 113-172. CMP 19:02

[P] D.A. Pelles (= D.A. Eisenman), Holomorphic maps which preserve intrinsic measure, Amer. J. Math., 97 (1975), 1-15. MR 51:3542

[PS] E.A. Poletsky, B.V. Shabat, Invariant metrics, Encyclopaedia of Math. Sci., 9(1986), 73125 (in Russian). English transl. in Encyclopaedia of Math. Sci., Vol.9. Several Complex Variables III. Berlin-Heidelberg-New York, Springer Verlag, 1989, 63-111. CMP 19:02

[Ra] V.V. Rabotin, A counterexample to two problems of Kobayashi, Multidimensional Complex Analysis (Russian), 256-257, Akad. Nauk SSSR, Sibirsk. Otdel., Inst. Fiz., Krasnoyarsk, 1985. MR 88i:32034

[Ro] H.L. Royden, Remarks on the Kobayashi metric. In Several Complex Variables, II (Maryland, 1970), Lect. Notes Math., 185(1971), 125-137. MR 46:3826

[S] Y.-T. Siu, Every Stein subvariety admits a Stein neighborhood, Invent. Math., 38(1976), 89-100. MR 55:8407

[T] R. Thom, Un lemme sur les applications differentiables, Bol. Soc. Mat. Mexicana (2), 1(1956), 59-71. MR 21:910

[W] H. Whitney, Complex Analytic Varieties, Addison-Wesley, 1972. MR 52:8473

Department of Mathematics \& Computer Science, University of Miami, Coral Gables, FLORIDA 33124

E-mail address: kaliman@paris-gw.cs.miami.edu

Université Grenoble I, Institut Fourier des Mathématiques, B.P. 74, 38402 Saint Martin d'Hères-CÉdex, France

E-mail address: zaidenbe@fourier.grenet.fr 